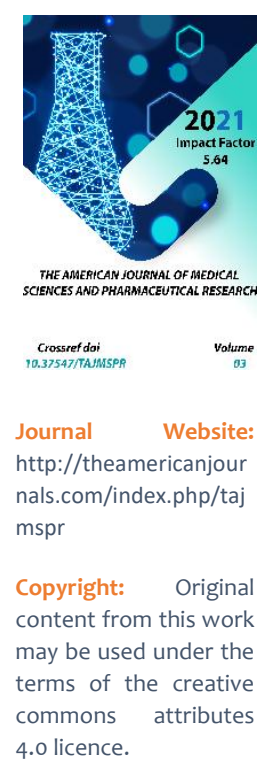

\title{
Peculiarities Of Treatment Of Purulent-Necrotic Complications In Patients With Diabetic Foot Syndrome
}

\author{
Abdullaev R.N. \\ Andijan State Medical Institute, Uzbekistan
}

\section{ABSTRACT}

The number of patients with diabetes mellitus in the Andijan region who were registered according to endocrinologists from 2000 to 2015 increased almost fourfold from 26,000 to more than 110,000 in 2016 (14). This also shows that the number of patients with various purulent-necrotic complications of diabetes mellitus also increases proportionally, in addition, there is an increase in patients with severe purulent-septic complications of these processes, with widespread, deep purulent-necrotic fasciitis [3]. Of patients in the department of surgical infection of the ASMI clinic, patients with purulent-necrotic complications of diabetes mellitus account for almost $78 \%$, which indicates a significant increase in the number of these patients. This tendency continues and goes up every year. According to the WHO, by 2030 every 15 people will have diabetes, and by 2050 this figure will be equal to every 6 people.

\section{KEYWORDS}

Purulent-Necrotic, tissues and tendon, WHO, antibiotic-resistant microflora.

\section{INTRODUCTION}

The clinical picture of purulent-necrotic complications in patients with diabetic foot syndrome is characterized by a peculiar course. This is due to ischemia of the affected area in varying degrees of severity, high bacterial contamination, with a tendency to spread the purulent process in the surrounding tissues and tendon sheaths and a significant decrease in the general and local reactivity of tissues $[1,3,4,8,13,17,18,19]$, 
which are pointed out by many authors who have established a violation of the cytokine background and beaten activity of protease in the wound - local factors slowing wound healing, also identified a systemic decrease, which is expressed in a decrease in the function of leukocytes and impaired peripheral circulation (15). To protect the body, scientists used drugs, physical effects, among which high-energy and low-energy lasers were used, were also used in the bloodstream, especially laser beams with 405 $\mathrm{nm}$, which stimulate the immune-competent structures of the body in the form of cytokine complexes $(12,15)$.

Considering the prevalence of antibioticresistant microflora in the wound, the increased frequency of seeding nonfclostridial anaerobic, conditionally anaerobic, and mixed infections $[6,7,8]$, the search for new methods and methods of treatment continues purulent wounds $[1,10,15,17,18,19]$

The urgency of the problem of local drug therapy of purulent-necrotic complications in patients with diabetic foot syndrome is due to the low efficiency of traditional means and methods of drug administration for the treatment of purulent process, the main disadvantage of which consists in the fact that they affect only individual components of the wound process $[5,9,13]$. Violation of blood flow through the capillaries and microcirculation disorders in $88 \%$ of patients with diabetes mellitus lead to changes in the endothelium of these vessels, leading to endothelium relaxation and a decrease in NO synthesis by endothelial cells, which leads to the development of endothelial edema, causing a tendency to thrombus formation. The hormone dexamethasone inhibits the development of such processes [1], and midocalm, no-shpa increases the blood supply to the limb and lowers peripheral resistance $[10,12,13,15]$.

The treatment of purulent-necrotic complications of diabetes mellitus is of particular importance given the fact that after radical, high amputations of the lower extremity, almost $90 \%$ of patients die within 2 years, and it is also necessary to perform a control amputation in half of the surviving patients $[7,8,12]$, while the method of choice is the development of new methods for limiting purulent-necrotic processes in the extremities and the use of organ-saving operations in this part of the body. These tasks are carried out with the use of a targeted effect of etiotropic (rational antibiotic therapy), pathogenetic (vasodilator, thrombolytic, anticoagulant, desensitizing preparations) factors in the area of the lower limb lesion (endoarterial).

The aim of this study was - to increase the effectiveness of treatment of purulentnecrotic complications of diabetic foot using methods and means that affect all links of the etiology and pathogenesis of the development of a purulent-necrotic process in the lower extremities in diabetes mellitus. This circumstance could not but affect the increase in the proportion of patients with decompensated forms of diabetes mellitus among all patients. The age of the patients ranged from 35 to 75 years. Men accounted for $71 \%$, women - $29 \%$. In many patients, concomitant diseases were identified, the leading place (65-72\%) among which was the pathology of the cardiovascular system, diseases of the liver, kidneys and lungs were often observed. Often, it was these concomitant diseases that determined the severity of the patient's condition and, in many cases, were the cause of their death. 
When seeding pus, bacteriological research in $87 \%$ of cases revealed aerobic-anaerobic associations, most often including obligateanaerobic non-spore-forming bacteria (Peptococcus SPP., Peptostreptococcus SPP., Bakteroides Fragile), facultative - anapaerobic ), anaerobic microorganisms (Pseudomonas aeruginosa). It should be emphasized that the high frequency (5-7\%) of excretion of Pseudomonas aeruginosa is probably due to long-term treatment of many patients in polyclinics and other hospitals.

The sensitivity of microorganisms to antibiotics was also determined. The greatest sensitivity was noted to the following antibiotics: gentamicin, cefataxime, ceftriaxone, sulbactamucuacef.

For the treatment of patients with purulentnecrotic complications of diabetic foot syndrome, a complex of drug treatment in the form of rational antibiotic therapy was used two types of synergistic antibiotics were used at the appropriate dose and interval, one antibiotic was administered intraarterially through the femoral artery once a day, together with $0,5 \%$ solution of novocaine, antispasmodics, thrombolytics and desensitizing hormone dexamethasone 0.3 $\mathrm{ml}$., This complex was proposed by us as a substitute for intra-arterial blockade according to Elansky, where the drug was used. In addition, the complex of therapy included immunotherapy, general desensitizing therapy, detoxification treatment and measures to restore impaired functions of organs and systems. We note the reaction of the tissues of the lower extremity to the intraarterial administration of the proposed complex of drugs was as follows: in patients with and after intra-arterial administration of the aforementioned complex of pharmacological agents, warmth was felt in the feet, in the lower leg, in the area of the knee joint and on the thigh. After three blockages in the area of a purulent-necrotic wound, its cleansing is noted with the development of fresh granulation tissue around and in the depth of the wound, pulsation appears on the projection of $A$. Dorsalis Pedis on the affected side and the pulsation gradually increases. The operations were carried out in a differentiated manner, depending on the nature of the pathological process. With the onset of gangrene, an attempt was made to conservative treatment, every opportunity was used to transfer it to dry. In the case of purulent-necrotic changes in the area of the toes, if necessary, after preliminary preparation, the foot was amputated or the toe was articulated, depending on the extent of the lesion. In case of failures and with a tendency to progression of the process in the proximal direction, a high amputation of the limb was performed (in 7 patients), from the lower leg in 4 patients, from the thigh in 3 patients.

As a result of using the proposed method of complex treatment of purulent-necrotic complications in diabetic foot syndrome, the number of high amputations decreased 5 times.

After opening the abscess and before starting treatment of the wound in patients with purulent-necrotic complications in the syndrome of diabetic foot was marked by pronounced local acidosis, reaching $\mathrm{pH}$ up to 5.0 - 5.2. This usually contributed to the expansion of the zone of tissue damage and maintained the activity of the infectious process. Considering the anti-inflammatory properties of a simple silk medium at pH 8.5, a $4 \%$ solution of soda was used topically to wash a purulent-necrotic wound. 


\section{THE RESULTS OBTAINED AND THEIR DISCUSSION}

In patients with diabetic foot, due to a pronounced disorder of the microvasculature, the growing microthrombosis prevents the full effect of drugs introduced into the general bloodstream on the area of the pathological focus. Based on this, in order to create a rational concentration of antibiotics in parts of the body affected by purulent-necrotic processes, as well as to restore local blood flow, in order to provide oxygen to regenerating cells, which are the basis for the regeneration of purulent-necrotic wounds, an intra-arterial method of introducing the proposed complex of drugs introduced through $A$.Femoralis of the affected side. Antibiotic therapy was used on the basis of the concept of rational antibiotic therapy, which indicates the use of at least two types of synergistic antibiotics in purulent-necrotic processes, one of which can be used for intraarterial administration. The recommended method was used in the form of a complex of drugs from antibiotics, thrombolytics, vasodilator, desensitizing and antiinflammatory drugs endoarterially. The manipulation was carried out for 5-7 days, once a day. We also used a local sorbent for detoxification, given that the bulk of patients with complicated diabetic foot are elderly and senile people with severe concomitant pathology, for detoxification of the body, the enterosorption method was used as a simple, generally available, non-invasive, at the same time highly effective way to combat with endogenous intoxication.Polyphepan was used as a sorbent at a daily dosage of $1.0 \mathrm{~g} / \mathrm{kg}$ of body weight for 8-9 days according to the method of Kh.T. Musashaikhov (5).

The reaction of the tissues of the lower extremity after intra-arterial injection of the proposed complex of drugs was such a feeling of warmth in the foot, in the lower leg, in the area of the knee joint and on the thigh immediately after the administration of the drugs. After three blockades, we managed to remove the affected limb from the state of critical ischemia, and in the area of the purulent-necrotic wound, its cleansing is noted with the development of fresh granulation tissue around and deep in the wound, pulsation appears on the projection of A. Dorsalis Pedis on the affected side. This complex of procedures was carried out in 100 patients, who subsequently underwent organsaving operations in the form of exarticulation, limited amputation of the toes of the foot, half of the foot - amputation of the metatarsal bones according to Sharp. Surgical tactics in the treatment of purulentnecrotic complications in diabetic foot syndrome consisted in carrying out economical, sparing methods of surgical treatment and debridement of the lesion focus, aimed at maximizing the preservation of the supporting function of the foot. As a result of using the proposed method of complex treatment of purulent-necrotic complications in diabetic foot syndrome, the number of high amputations decreased 5 times.

After opening the abscess and before starting treatment of the wound in patients with purulent-necrotic complications in the syndrome of diabetic foot was marked by pronounced local acidosis, reaching $\mathrm{pH}$ up to 5.0 - 5.2. This usually contributed to the expansion of the zone of tissue damage and maintained the activity of the infectious process. Considering the anti-inflammatory properties of a simple silk environment at a $\mathrm{pH}$ of 8.5 , a $4 \%$ solution of soda was used topically to wash a purulent-necrotic wound.

Relief of pain and reduction of edema, tissue infiltration around the wound gradually 
subsides, and the amount of purulent discharge sharply decreases. On days $5.8+$ 0.76 , the wound was usually cleared of necrotic tissue. On days $7.3+0.57$, juicy, grainy, easily bleeding granulations appeared.

The use of a complex of drug treatment in the form of rational antibiotic therapy, in which the antibiotic was also administered intraarterially through the femoral artery once a day, together with a $0.5 \%$ solution of novocaine, antispasmodics, thrombolytics and the hormone dexamethasone $0.3 \mathrm{ml}$., In addition, in the complex therapies included immunotherapy, desensitizing therapy, detoxification treatment, and measures to restore impaired functions of organs and systems. As a result of the work carried out, $86 \%$ of patients achieved preservation of the supporting function of the limb with the use of organ-preserving operations on the limb. Amputations at the hip level were performed only in 3 patients (3\%), at the level of the lower leg - in 4 (4\%), at the level of the foot - in 32 (32\%), exaarticulations of the fingers were performed in 31 (31\%), necrosectomy - in 21 (21\%) patients, in 7 patients, the necrotic process on the toes was self-rejected. Mortality among patients was not observed.

The average length of stay of patients in the hospital was $11.5+2.4$ days

\section{CONCLUSION AND CONCLUSIONS}

The obtained data confirm that: $\{\{1\}\}$

1. Use of the proposed complex of drug treatment in the form of rational antibiotic therapy, in which the antibiotic was also administered intra-arterially through the femoral artery once a day, together with $0.5 \%$ novocaine solution, antispasmodics, thrombolytics and the hormone dexamethasone $0.3 \mathrm{ml}$., Purulent -necrotic lesions of the lower extremity in patients with diabetic foot syndrome allows a radical decrease in purulent-necrotic changes in the lower extremity, which allows the treatment of these changes by organ-preserving methods of surgery, without resorting to high amputations.

2. It is advisable to use immunotherapy, desensitizing therapy, detoxification treatment and measures to restore impaired functions of organs and systems in the complex of therapy, which allows to improve the results of treatment of purulent-necrotic complications in diabetic foot syndrome

\section{REFERENCES}

1. Abdullaev RN / Complex treatment of purulent-necrotic lesions of the lower extremity in patients with diabetic foot syndrome. // New day in medicine. 2021 No. 2 (34), p.413-417.

2. Abdullaev R.N., Yusupov K.A., Abdullaeva M.A. et al. / Application of prolonged action enzymes impregnated on synthetic materials for cleansing necrotic wounds. // In the collection Actual problems of modern medicine. Medicine today, tomorrow. Republican conference with international participation. 2018. pp. 603$604 \cdot\{\{1\}\}$

3. Abdullaev S.A., Musaev S.T. / Surgical treatment of necrotizing fasciitis in patients with diabetes. // Collection of scientific papers Diabetes mellitus, its complications and surgical infections. Practical Congress, Moscow, 2019. November 19-21. Moscow. Publishing "Pero" -108 p.3-4.

4. Babadzhanov B.D., Islamov M.S., Zhanabaev B.B. et al. / Application of longterm intra-arterial catheter therapy in the treatment of purulent-necrotic lesions of 
the foot in patients with diabetes mellitus // Pathology. -2000.- № 4.- P. 52-54.

5. Bazlova SB / Complex treatment of purulent-necrotic lesions of the lower extremity in patients with diabetes mellitus against the background of decompensated ischemia. // Successes of modern natural science. 2005. No. 9. Pp. 89-90.

6. Belyaeva OA, Radzikhovsky AP, Krizhevsky et al. / Results of studies of the degree of ischemia in diabetic foot. // Academy of electr. Publications. Kiev. 2012 No. 1 (87) 1P. P.L. Shupik National Medical Academy of Postgraduate Education.

7. Musashaikhov Kh.T., Ikramova F.D., Vasilevsky E.A. and etc./ Complex treatment of patients with purulentnecrotic lesions of the foot in patients with diabetes mellitus. // Tibbiyotda yangi kun 2018 \# 3.455-461

8. Tarakanova OE / Complex treatment of patients with purulent-necrotic complications of diabetic foot syndrome. // Dissertation for the degree of candidate of medical sciences, Nizhny Novgorod 2008, 122 pages.

9. Tarakanova O.E., Smirnov I.F. / Nonclostridial anaerobic infection in the purulent department of the city hospital. // Wounds. Wound infection. Mat. 1V AllRussian Conference of General Surgeons with International Participation and the Plenum of the Problem Commission "Infection in Surgery". Yaroslavl, 2007. pp. 183-185.

10. Tarakanova OE, Kurykin MI, Kleshkin A. E. I A new approach to the treatment of chronic arterial insufficiency. // Outpatient surgery No. 28.20076, p. 116.

11. Tartakovsky EA, Gvozdev NA. et al. Treatment of complications of "diabetic foot" // Surgery. - 2009. - No. 10. - P. 53-55.

12. Tolstykh P.I., Ivanyan A.N., Geynits A.V. et al. // Little secrets of the use of laser radiation in the prevention and treatment of purulent wounds. M., $200959 \mathrm{pp}$.

13. Prokhorov A.V., Dushkevich V.S. Treatment of wet foot gangrene in patients with diabetes mellitus // Surgery. - 2011. - N7. - S. 11-14.

14. Khatamov E.A., Khatamov M.E. / Peculiarities of studying the epidemiology of patients with diabetes mellitus in the Andijan region. // Materials of the Republican conference of endocrinologists of Uzbekistan with international participation. Tashkent city, 2017 P.96-97.

15. Shiryaev V.S. Bugrovsky O.N., Shvetsky F.M. et al. / Treatment of purulent-necrotic processes with the use of intravenous laser region. blood (405 nm.). // Collection of scientific tr.Diabetes mellitus, its complications and surgical infections. Materials of the 4th International Scientific and Practical Congress. Moscow, 2019. 1921 November. Moscow. Publishing "Pero" 108p. 96-99p.

16. Chur N.N. / Treatment of patients with chronic lower limb ischemia in diabetes mellitus. // Surgery News, 2008, No. 16 (1), p.61-66.

17. Hitoshu Nukada / Diabetic Microangiopathy - an overview I ScienctDirect Topics - /I Amer.J.Med. - 2006. - Vol.85, N5A. - p. 119130.

18. Camera A. / Diabetic Microangiopathy: physiopatological, clinical and therapeutic aspectis // Minerva Endocrinolog. 2007. sep. Pub / Med.

19. Wagner F.W. The diabetic foot.//Ortopedics.-2005.-N 10.-p.163-171. 